\title{
Responses of the Normal Human to Infusions of Plasma from Patients with Graves' Disease*
}

\author{
Claude D. Arnaud, $\dagger$ Hans A. Kneubuhler, $\$$ Virginia L. Seiling, \\ Bruce K. Wightman, and Norman H. Engbring \\ (From the Department of Medicine, Marquette University School of Medicine and Milwaukee \\ County General Hospital, Milwaukee, Wis.)
}

The cause of the hyperthyroidism of Graves' disease is unknown. Ever since Loeb and Bassett (1) and later Siebert and Smith (2) showed that hyperthyroidism could be produced in animals by injections of pituitary extracts, it has been suggested that excess secretion of thyrotropin (TSH) is responsible for this disease in man. However, the bioassay of TSH in the blood of patients with Graves' disease has led to inconsistent results (3). Until recently, direct experimental support of the concept of endogenous overstimulation of the thyroid gland has been lacking. The studies of Adams and Purves (4-8) and of McKenzie (9-11) have shown that the serum of many patients with Graves' disease contains a factor that stimulates the thyroid glands of mice. This factor is different from bovine TSH in that it has a longer biologic "half-life" (11) and a delayed time of action $(7,9)$ and is not inactivated by TSH antisera (12). The site of production of this thyroid stimulator is not known, but there is negative evidence which suggests that the pituitary is not its source $(11,13)$. There is a close correlation between the presence of this thyroid stimulator and active Graves' disease, but the question of its

* Submitted for publication November 20, 1964 ; accepted April 8, 1965.

This investigation was supported by U. S. Public Health Service grants 5T1 AM 5023-10, AM 04672, AM $01200-12,5$ MOL FR-00058-04, and 1-GS-134 and by American Medical Association grant-in-aid 482.

Presented in part at the meeting of the Midwestern Section of the American Federation for Clinical Research, October 1962.

$\dagger$ Work done during tenure as U. S. Public Health Service trainee in metabolism. Address requests for reprints to Dr. Claude D. Arnaud, Dept. of Biochemistry, University of Wisconsin, Madison, Wis.

\$Work done during tenure as U. S. Public Health Service trainee in metabolism. Present address: V.A. Hospital, Wood, Wis. role in the production of the hyperthyroidism of this disorder remains unanswered (11).

The present investigations were designed to study the effect of the infusion of whole plasma obtained from patients with Graves' disease on the organic iodine metabolism of normal man as measured by changes in endogenously synthesized organically bound iodine in the blood of recipients. A sensitive model for the study of thyroid-stimulating substances in man was used and is described. The results indicate that there is a factor in the plasma of some of the patients with Graves' disease studied which stimulates organic iodine metabolism in normal man. Furthermore, the comparative patterns of response to 1) various doses of bovine TSH, 2) plasma from a patient with spontaneous myxedema (myxedema plasma), and 3) plasma from patients with Graves' disease (Graves' plasma), who were hyperthyroid, hypothyroid, and euthyroid at the time of donation, indicate that there are qualitative differences between the effects of commercial bovine TSH and endogenously produced TSH and those of the thyroid-stimulating principle in Graves' plasma.

\section{Methods}

Recipients. All recipients were male volunteers between the ages of 40 and 60 years. None had a personal or family history of thyroid or hepatocellular disease nor had they been taking any drug or iodine-containing materials. Physical examination was normal except for mild hypertension in one, and findings were consistent with mild chronic bronchitis in another. They were euthyroid as evidenced by clinical evaluation by two of us, protein-bound iodine (PBI) concentration of serum, and 24-hour thyroidal accumulation of $\mathrm{I}^{182}$. They were hospitalized on a metabolic ward during the entire period of study.

Donors. Case summaries of the donors are contained in the Appendix. An outline of the pertinent manifestations of their diseases and the recipient of each plasma are shown in Table I. Donors of normal plasma were 


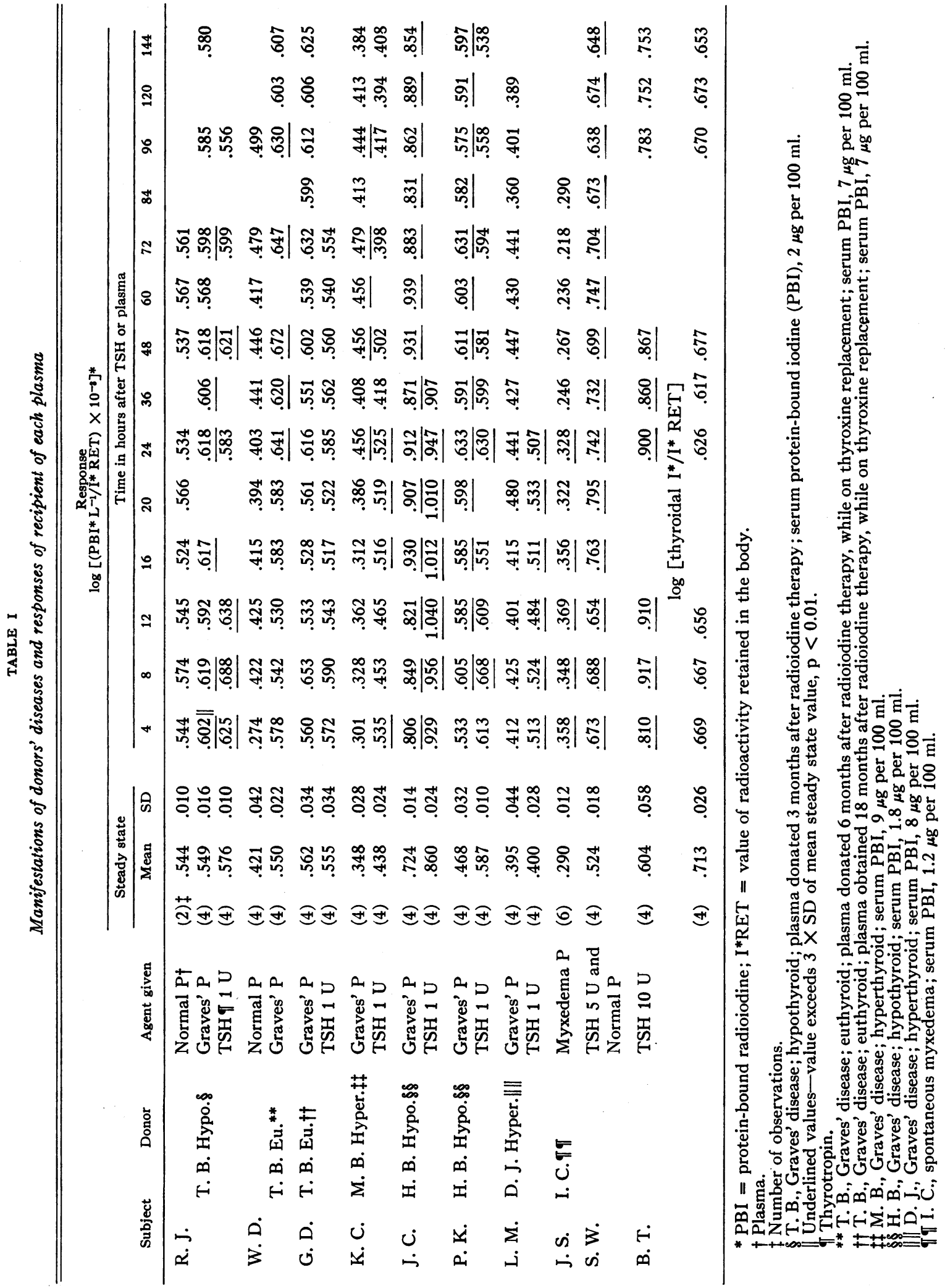


young healthy males without personal or family history of thyroid disease, and the concentrations of proteinbound iodine of these plasmas were within normal range.

Plasma. Plasma was either frozen or lyophilized immediately after it had been donated. It was studied for its serological reaction for syphilis and its titer of antibodies to $\mathrm{A}, \mathrm{B}$, and $\mathrm{Rh}$ red blood cell antigens. None of the donors had a personal or family history of liver disease nor was enlargement or tenderness of the liver detected on physical examination. Just before infusion, plasmas were either thawed slowly or reconstituted with sterile distilled water and studied with respect to their ability to agglutinate the proposed recipient's red blood cells. Judged by these criteria, all plasmas that were infused were considered safe.

Isotope technique. The relative quantity of serum from patients with Graves' disease that has been reported to produce maximal increases in the blood radioactivity of mice whose thyroid glands had been labeled with $I^{131}$ is greater than that which could be conveniently obtained from donors and safely infused into humans. This made it necessary to utilize a study model in which small changes in the endogenously synthesized organic iodine in plasma could be precisely measured. An isotopic technique was adapted in part from that described by Berson and Yalow (14), the sensitivity of which depends on relating the concentration of the organically bound radioiodine in the blood to the total body radioiodine pool at the time of blood sampling.

Carrier free $\mathrm{I}^{121}$ or $\mathrm{I}^{125}\left(\mathrm{I}^{*}\right)$ as sodium iodide was given to subjects either intravenously or orally in a single dose of $200 \mu \mathrm{c}$. Urine was collected thereafter for periods terminated by times of blood sampling. Whenever possible, blood was obtained every morning and at appropriate intervals after the administration of TSH or plasma. Thyroidal radioactivity was assayed with a 2 -inch crystal scintillation detector at the time of blood sampling. Stools were collected and incinerated to ash. An automatic well-type scintillation spectrometer was employed for assays of radioactivity in blood and excreta.

Protein-bound radioiodine ( $\mathrm{PBI} \mathrm{I}^{*}$ ) in the plasma was determined in duplicate as the difference between the radioactivity assayed in $3 \mathrm{ml}$ of whole plasma and that in a $30 \%$ trichloroacetic acid extract of $3 \mathrm{ml}$ of plasma after appropriate correction for change in volume. $\mathrm{Ra}$ dioactivity in the urine was assayed on duplicate $3-\mathrm{ml}$ volumes of a well-mixed sample. Three-ml weighed volumes of stool ash were counted, and the radioactivity was related to the total weight of the specimen. Only studies in which plasma levels of radioactivity exceeded 5 times background are reported, and the counting error of all assays never exceeded $10 \%$.

All data were corrected for radioactive decay. $\mathrm{Cu}$ mulative losses of radioactivity in the urine samples were subtracted from the amount originally administered, and a value of radioactivity retained in the body (I* RET) coincident with blood sampling was obtained. Radioactivity excreted in the stool was found to be negligible after the first 7 days and was disregarded in all calculations because infusion and drug studies were always per- formed after day 12. The plasma concentration of PBI* was related to the size of the existing iodine pool and was expressed as the ratio PBI* per liter to the total I* retained ( $P B I^{*} L^{-1} / I^{*} R E T$ ). Thyroidal radioactivity was expressed in a similar manner (thyroidal I*/I* RET).

Plasma and drug administration. Neither exogenous thyroid hormone nor antithyroid agents were given to subjects as has been reported in animal studies (15). In one study, $250 \mathrm{ml}$, and in all others, $500 \mathrm{ml}$ of plasma was given intravenously over a period of 1 hour. The authors recognized the desirability of administering $\mathrm{TSH}^{1}$ intravenously in plasma, but adverse reactions to the drug given by this route (16) prompted intramuscular injection. TSH and plasma were administered only during the steady state period (see Results).

Experiments were originally designed so that subjects would act as their own controls and receive in random successive order: Graves' plasma, normal plasma, and $1 \mathrm{U}$ of TSH. This plan was altered after the results of two normal plasma infusions were known, and it was felt that additional studies with normal plasma would not yield any further significant information and could not be justified.

Data. In evaluating responses it was found that the logarithmic transformation of the data allowed more valid comparison of the magnitudes of the various responses. Since the pattern of response varied with the agent administered, a valid statistical criterion of comparison could not be employed. However, within an experiment the period of response was arbitrarily considered that in which values for the $P B I^{*} / I^{*}$ RET exceeded 3 times the standard deviation from the mean of at least 4 values obtained during the control period immediately preceding drug administration $(p<.01)$ (17).

In viewing the response curves, significance was attached only to the curve as a whole rather than to the individual points of which it was composed. Any attempt to draw conclusions from a few points that did not fit the general contour of the curve was felt to be too speculative and unwarranted.

\section{Results}

Study model. The values of the ratios PBI* $\mathrm{L}^{-1} / \mathrm{I}^{*} \mathrm{RET}$ and thyroidal I*/I* RET achieve an approximately horizontal asymptote 10 to 14 days after administration of radioiodine. There were no exceptions to this pattern, and these results are similar to those obtained by Berson and Yalow (14) in euthyroid subjects. In a few potential subjects the quantity of precipitable radioactivity of the plasma during the steady state period was too low for accurate assay. These subjects had low normal values for the per cent 24-hour accumulation of radioiodine. The results of studies

1 Thytropar, bovine thyrotropin, Armour Pharmaceutical Co., Kankakee, IIl. 
done with $\mathrm{I}^{125}$ were similar to those done with $\mathrm{I}^{131}$ except that the longer half-life of $\mathrm{I}^{125}$ made it possible to study an individual subject for a longer period of time. Steady state values of the ratio $\mathrm{PBI}^{*} \mathrm{~L}^{-1} / \mathrm{I}^{*} \mathrm{RET}$ varied little (mean standard error of 4 determinations before administration of a drug in all studies $\pm 4.0 \%$ ). The sensitivity of this system progressively diminished with time as the levels of radioactivity declined.

Studies with TSH and myxedema plasma. TSH was studied at three dose levels: 1.0, 5.0, and $10.0 \mathrm{U}$. One unit elicited similar responses in five of seven subjects. The PBI* $\mathrm{L}^{-1} / \mathrm{I}^{*} \mathrm{RET}$ increased rapidly from steady state levels to peak values within the first 20 hours and then decreased to one-half of the peak value within 24 to 36 hours (Figure 1A). The response obtained with $10 \mathrm{U}$ of TSH (Table I, subject B.T.) was similar to that obtained with $1 \mathrm{U}$ with respect to the time of attainment of peak values, but the amplitude and duration of response were proportionately greater. Five units of TSH was administered intramuscularly at the same time as the infusion of $500 \mathrm{ml}$ of normal plasma in subject S.W. (Table I). Although the pattern of response was similar to those obtained when TSH was given without concurrent plasma infusion, the peak response was slightly delayed. There was no change in the thyroidal I*/I* RET after $1 \mathrm{U}$ of TSH in one subject so studied, but after $10 \mathrm{U}$ in subject B.T., this index decreased slowly, reached a minimum at 36 hours, and then rose to a new steady state level (Table I).

The response obtained following the infusion of $500 \mathrm{ml}$ of myxedema plasma into subject J.S. is illustrated in Figure 1B. It is remarkably similar to the one shown above in Figure 1A, which was obtained with $1 \mathrm{U}$ of TSH.

Studies with Graves' plasma. Plasma was obtained from four patients with Graves' disease, one of whom donated blood while hypothyroid after radioiodine therapy and while euthyroid on thyroxine replacement. These plasmas were infused into seven subjects and elicited responses that were considered positive in five. Of the two experiments in which no response was observed, one subject responded typically to subsequently administered TSH (L.M.) and the other (G.D.) did not (Table I). The subject who responded to $\mathrm{TSH}$ received plasma (D.J.) that had not been

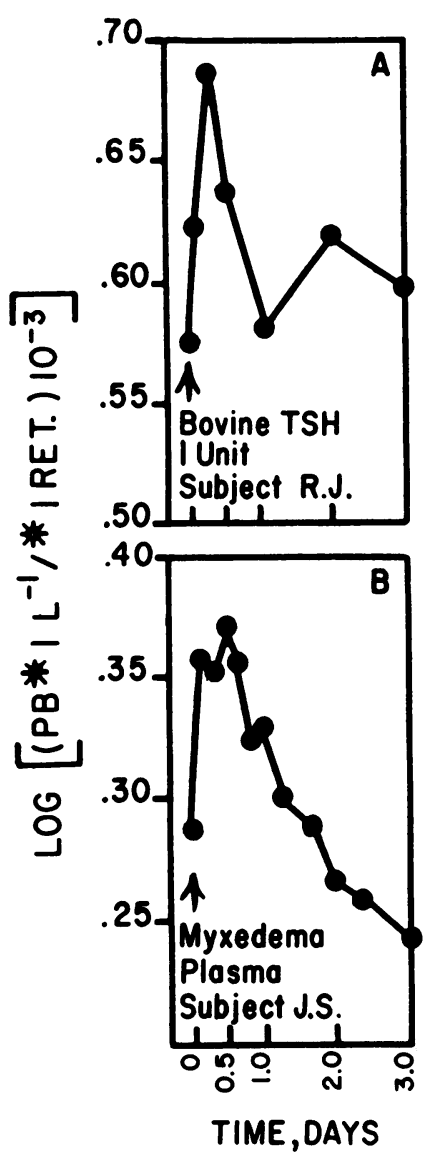

Fig. 1. REsponses to thyrotropin (TSH) and MYXEDEMA PLASMA. Response of subject R.J. to $1 \mathrm{U}$ of bovine TSH (A) and subject J.S. to the infusion of 500 $\mathrm{ml}$ of plasma donated by a patient with spontaneous myxedema (B) as measured by changes in endogenously synthesized organically bound iodine in the blood of the recipients. TSH or plasma was administered at zero time after stabilization of the base line. $\mathrm{PB} * \mathrm{I}=$ proteinbound radioiodine; $* \mathrm{I}$ RET $=$ radioactivity retained in the body.

previously studied, but the subject who did not respond to TSH received plasma from the donor (T.B.) whose plasma had given positive responses (W.D. and R.J., Figure 2, A and B) 1 and 1.5 years before.

The times of the maximal responses to Graves' plasma were delayed, and the durations of the responses were prolonged when compared with those obtained with $1 \mathrm{U}$ of TSH or myxedema plasma. The amplitudes of responses elicited by infusions of Graves' plasma were either equal to or slightly less than those elicited by companion TSH responses. However, two distinct types of response 


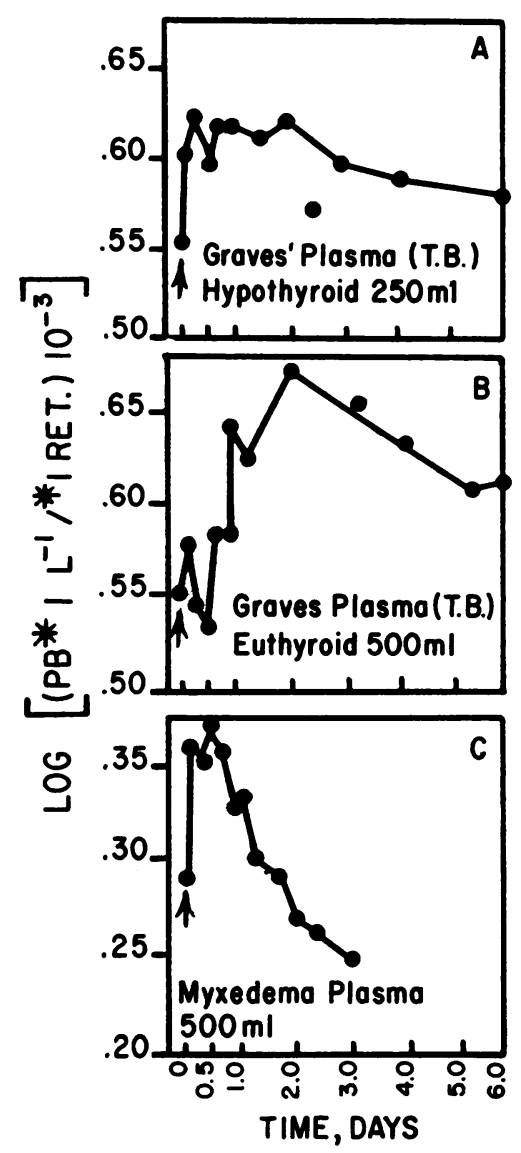

Fig. 2. Comparison of the responses to Graves' and MYXEDEMA PLASMa. Response of subject R.J. to the infusion of $250 \mathrm{ml}$ of plasma donated by T.B. while the latter was hypothyroid (A) and of subject W.D. to 500 $\mathrm{ml}$ of plasma donated by the same patient when he was euthyroid (B) as measured by changes in endogenously synthesized organically bound iodine in the recipient's blood. (C) Figure 1B for comparison.

were observed after Graves' plasma infusions. The results of three experiments done in different subjects are compared in Figure 2, A, B, and C. The Graves' plasmas used in studies A and B were obtained from the same patient (T.B.), but the plasma used in study A was donated during a period of hypothyroidism induced by radioiodine (PBI, $2 \mu \mathrm{g}$ per $100 \mathrm{ml}$ ), and the plasma in study $B$ was donated after the patient had been euthyroid (PBI, $7 \mu \mathrm{g}$ per $100 \mathrm{ml}$ ) for 3 months. A clear difference is apparent in the time of the maximal response. The hypothyroid Graves' plasma elicited a maximal response within the first 12 hours as did the plasma from the patient with spon- taneous myxedema (study C); but no response was observed after infusion of euthyroid Graves' plasma until 24 hours had elapsed, and peak values were attained only after 48 hours. The infusion of $250 \mathrm{ml}$ and $500 \mathrm{ml}$ of normal plasma into these subjects (R.J. and W.D., respectively) elicited no response (Table $\mathrm{I}$ ).

In an attempt to confirm these observations, two studies were done with plasma obtained from H.B. before he was treated for severe hypothyroidism and one with plasma from M.B., who had a mild recrudescence of hyperthyroidism. Similar results were obtained (Table I). The hypothyroid Graves' plasma elicited a combined (early and delayed) response and the hyperthyroid Graves' plasma only a delayed response. No change in thyroidal I*/I* RET was observed after Graves' plasma infusion in subjects R.J. and W.D.

McKenzie assay. Assay of $0.5 \mathrm{ml}$ of serum from H.B. in the McKenzie mouse assay system for the long-acting thyroid stimulator (9) showed an increase in blood radioactivity of $800 \%$ above control levels at 9 hours and $200 \%$ at 3 hours. Assays on other plasmas were not available.

\section{Discussion}

It is apparent from these studies that plasma donated by three of four patients with Graves' disease contained a factor that stimulated organic iodine metabolism in normal man in a manner distinctly different from bovine TSH or the thyroid stimulating material in myxedema plasma. There is no direct evidence in the present report which indicates that the thyroid glands of the recipients were the sources of the increased concentrations of organically bound iodine in the blood that were detected following the infusion of Graves' plasma. However, the studies of McKenzie (10) have shown histologic changes consistent with thyroidal overactivity in mice given repeated injections of sera known to have long-acting stimulator activity, and it is reasonable to assume that either direct or indirect stimulation of the thyroid gland occurred in the present studies.

The infusion of normal plasma probably altered the response pattern observed after the administration of thyroid stimulating materials. As shown in Figure 1, A and B, the peak response observed after $500 \mathrm{ml}$ of myxedema plasma is less sharp than that obtained with intramuscular administration 
of $1 \mathrm{U}$ of TSH. This suggests that dilution of the intravascular pool of protein-bound radioactivity may have obliterated a portion of this response. The slight delay in the attainment of the peak response observed after the concurrent administration of $5 \mathrm{U}$ of TSH and $500 \mathrm{ml}$ of normal plasma (subject S.W., Table I) also suggests this. On the other hand, the responses obtained with either $1 \mathrm{U}$ of TSH or myxedema plasma had subsided to less than half the maximal amplitudes within 36 hours of administration. Any response to plasma infusion that might occur after that time is not likely to be an artifact of volume expansion.

If the response observed after the infusion of myxedema plasma reflects endogenously produced $\mathrm{TSH}$, its effect in man is quite similar to that of commercial bovine TSH. Using the average total amplitude of response obtained in five subjects as a standard response to $1 \mathrm{U}$ of bovine $\mathrm{TSH}$, a gross estimation shows that this plasma contained between 1 and $1.5 \mathrm{mU}$ per $\mathrm{ml} \mathrm{TSH}$. This figure is in good agreement with the high concentrations of $\mathrm{TSH}$ that have been reported by a number of investigators to be present in the plasma of myxedematous patients $(3,18)$.

The two patterns of response induced by the infusion of Graves' plasma (Figure 2, A and B) that appear to correlate with the level of circulating thyroxine are of interest. Hyperthyroid and euthyroid Graves' plasma elicited no response during the first 24 hours after infusion, but a rapid increase of the PBI* $\mathrm{L}^{-1} / \mathrm{I}^{*} \mathrm{RET}$ occurred within 4 to 8 hours after hypothyroid Graves' plasma. If the former reflects levels of endogenous TSH too low to measure with the technique employed, and the latter, greater than normal concentrations of $\mathrm{TSH}$, support is given to the idea that the prolonged responses which were obtained with both plasmas after 24 hours were separate and distinct, and were elicited by a thyroid-stimulating substance peculiar to Graves' plasma. A critical study designed to analyze the temporal relationships between the responses to hyperthyroid, euthyroid, and hypothyroid Graves' plasma in the McKenzie mouse system should be helpful in the further evaluation of these observations. The results of the present studies, however, strongly suggest that in Graves' disease, the TSH secreting mechanism of the pituitary is subject to the usual negative feedback control related to blood thyroxine concen- tration, but production of the thyroid stimulator peculiar to Graves' disease is not. Since the responses evoked in these studies in humans are similar to those observed in animals, it is presumed that the responsible component is the long-acting thyroid stimulator (LATS).

Björkman, Denneberg, and Hedenskog studied the effect of the transfusion of $150 \mathrm{ml}$ of blood obtained from patients with Graves' disease on the $\mathrm{PBI}$ in the blood of recipients who required transfusion (19). They showed a consistent increase in the PBI after the infusion of euthyroid or hyperthyroid Graves' blood into four of eight recipients, with maximal values attained between 30 and 40 hours. A single study in which the responses to the intramuscular injection of 20 and $40 \mathrm{U}$ of U.S.P. TSH were compared with that obtained with the transfusion of Graves' blood showed 1) a similar time course of response of the PBI to TSH and the transfusion of Graves' blood and 2) a response to Graves' blood transfusion almost as great as the response to $20 \mathrm{U}$ of $\mathrm{TSH}$. In their studies, blood samples were not obtained between 6 and 24 hours (Figure 1A). It is difficult to evaluate the second finding of these workers; however, the most plausible explanation is that they compared the declining portion of the responses to large doses of TSH with those obtained with Graves' blood.

Spontaneous Graves' disease has not been described in species other than man. The studies recorded in this paper leave little doubt that the factor in Graves' plasma which stimulates the thyroid gland of the mouse does so in the human and in a manner qualitatively different from bovine or human TSH. At the time that these studies were done a method for the concentration of LATS from plasma (20) was not generally available. Further studies with the purified material in man, using the technique described, should be of considerable interest.

\section{Summary}

1) A sensitive method for the study of thyroid stimulating materials in man is described.

2) One unit of intramuscularly administered bovine thyrotropin (TSH) induced a rapid increase in endogenously synthesized organically bound iodine in the blood of 5 of 7 recipients. 
The responses reached a peak within 20 hours and had decreased to less than half the peak value within 24 to 36 hours. Ten units of bovine TSH elicited a pattern of response similar to that with $1 \mathrm{U}$, but the duration and amplitude of response were considerably greater. Concurrent infusion of $500 \mathrm{ml}$ of normal plasma delayed by several hours the apparent peak response to $5 \mathrm{U}$ of bovine TSH.

3) The infusion of $500 \mathrm{ml}$ of plasma from a patient with spontaneous myxedema elicited a response similar to that observed with $1 \mathrm{U}$ of bovine TSH.

4) Two infusions of normal plasma into two subjects elicited no response.

5) Plasmas obtained from four patients with Graves' disease, with varying states of thyroid function, were infused into seven normal recipients. Both immediate and prolonged responses were observed with hypothyroid Graves' plasma but only a delayed, prolonged response with euthyroid or hyperthyroid Graves' plasma.

6) It is concluded that there is a factor in the plasma of some patients with Graves' disease that stimulates organic iodine metabolism in normal man. This stimulator (presumably long-acting thyroid stimulator) is different from bovine TSH and the thyroid stimulating material (presumably $\mathrm{TSH}$ ) in plasma from a patient with spontaneous myxedema.

\section{Acknowledgments}

We thank Dr. Tibor Greenwalt, director of the Milwaukee Blood Center, and Dr. Shirley Johnson for their advice and invaluable aid in preparing plasmas for administration. Our sincerest appreciation goes to $\mathrm{Dr}$. Brian Webster, Thyroid Research Laboratory, Toronto General Hospital, for sending us the plasma of patient H.B. for study and the McKenzie mouse assay done on this plasma. We are most grateful to the staffs of the Marquette Clinical Research Center and the Milwaukee County Hospital Radioisotope Department for technical aid. We are indebted to Dr. William W. Engstrom, who has been a constant source of stimulation and helpful criticism.

\section{Appendix}

\section{Case summaries on plasma donors}

Patient T.B., Graves' disease. A 53-year-old white male first noted "bulging of eyes," blurred vision, and excessive tearing in 1954. In 1956 he developed increased appetite, weight loss, increased tolerance for cold weather, insomnia, and diplopia. Symptoms progressed causing him to stop work until he was treated at another hospital in 1959 with a "radioactive pill." His health improved, but symptoms recurred early in 1961. At that time he had diffuse thyroid enlargement, exophthalmos, fine tremor of the hands, and rapid atrial fibrillation. Protein-bound iodine (PBI) was $15 \mu \mathrm{g}$ per $100 \mathrm{ml}$, and thyroidal accumulation of $\mathrm{I}^{182}$ was $58 \%$ of the orally administered dose. He was treated with reserpine and $7 \mathrm{mc}$ $\mathrm{I}^{132}$. Three months later he developed signs and symptoms of mild hypothyroidism (PBI, $2.0 \mu \mathrm{g}$ per $100 \mathrm{ml}$; uptake of $\mathrm{I}^{281}$ by the thyroid was $6.0 \%$ ). Plasma was obtained for study in subject R.J. Thyroxine was given in gradually increasing amounts to $0.3 \mathrm{mg}$ daily, and after the PBI remained in the range of 6.5 to $7.0 \mu \mathrm{g}$ per $100 \mathrm{ml}$ for 3 months, plasma was drawn for study in subject J.S. and again in 12 months for study in subject G.D.

Patient M.B., Graves' disease. A 37-year-old white female had had "bulging eyes" for at least 10 years. In 1959 she developed a goiter, excessive sweating, heat intolerance, fatigue, palpitation, and nervousness, and a subtotal thyroidectomy was performed. Exophthalmos persisted, but she was otherwise well until October 1962, when hyperthyroidism recurred. PBI was $8 \mu \mathrm{g}$ per 100 $\mathrm{ml}$, thyroidal accumulation of $\mathrm{I}^{181}$ was $51 \%$, and this value was suppressed only to $41 \%$ after 1 week of triiodothyronine, $25 \mu \mathrm{g}$ four times daily. Plasma was drawn soon afterwards and studied in subject K.C. She was treated with $8 \mathrm{mc}$ of $\mathrm{I}^{131}$, and symptoms gradually abated.

Patient D.J., Graves' disease. A 33-year-old Negro female suffered gradually progressive tremor of the hands, nervousness, insomnia, palpitations, excessive sweating, increasing appetite, and weight loss for 8 months. She had a goiter with a systolic bruit, tachycardia, pretibial myxedema, but no exophthalmos. PBI was $21.7 \mu \mathrm{g}$ per $100 \mathrm{ml}$, and thyroidal accumulation of radioiodine was $72 \%$. Plasma was drawn for study in subject L.M. She was treated successfully with propylthiouracil and then subtotal thyroidectomy.

Patient H.B., Graves' disease. A 55-year-old white male developed clubbing of fingers in 1945 at which time he was euthyroid. He suffered rapid onset of weight loss (50 lb), tremor, excessive sweating, and heat intolerance, but no exophthalmos, in 1952. Between 1952 and 1953 he was given $20 \mathrm{mc}$ of $\mathrm{I}^{131}$ in 3 divided doses. Three months after his last dose he noted diplopia, which was followed by exophthalmos. Between 1954 and 1955 he suffered recurrence of hyperthyroidism, which was treated successfully with propylthiouracil, but he developed pretibial myxedema, which was confirmed by biopsy. He was first seen by Dr. Brian Webster in 1962 at which time he had extensive pretibial myxedema, severe exophthalmos (O.D. $29 \mathrm{~mm}$, O.S. $30 \mathrm{~mm}$ ), moderate ophthalmoplegia, periorbital edema, clubbing of fingers and toes, and myotonic deep tendon reflexes. Protein-bound iodine was $1.8 \mu \mathrm{g}$ per $100 \mathrm{ml}$, and thyroidal accumulation of $\mathrm{I}^{181}$ was $7.0 \%$. Plasma was drawn at that time for study in subjects J.C. and P.K. 
Patient I.C., spontaneous myxedema. A 44-year-old white female developed slowly progressive symptoms of hypothyroidism about 12 years previously. These included cold intolerance, dry skin, lethargy, deepening of the voice, and puffiness about the eyes. She demonstrated the typical myxedematous features including myotonic tendon reflexes. There was no palpable thyroid tissue. Serum PBI was $1.5 \mu \mathrm{g}$ per $100 \mathrm{ml}$, and thyroidal uptake of radioiodine was $2.0 \%$ at 24 hours with no increases after thyrotropin stimulation. Plasma was obtained for study in subject J.S. before the patient was treated.

\section{References}

1. Loeb, L., and R. B. Bassett. Effect of hormones of anterior pituitary on thyroid gland in the guinea pig. Proc. Soc. exp. Biol. (N. Y.) 1929, 26, 860.

2. Siebert, W. J., and R. S. Smith. The effect of various anterior pituitary preparations upon basal metabolism in partially thyroidectomized and in completely thyroidectomized guinea pigs. Amer. J. Physiol. 1930, 95, 396.

3. McKenzie, J. M. Bio-assay of thyrotropin in man. Physiol. Rev. 1960, 40, 398.

4. Adams, D. D., and H. D. Purves. Abnormal responses in the assay of thyrotrophin. Proc. Univ. Otago Med. Sch. 1956, 34, 11.

5. Adams, D. D. The clinical status of patients whose sera have given the abnormal response when assayed for thyrotrophin. Proc. Univ. Otago med. .Sch. 1956, 34, 29.

6. Adams, D. D., and H. D. Purves. The role of thyrotrophin in hyperthyroidism and exophthalmos. Metabolism 1957, 6, 26.

7. Adams, D. D. The presence of an abnormal thyroidstimulating hormone in the serum of some thyrotoxic patients. J. clin. Endocr. 1958, 18, 699.

8. Adams, D. D. Bioassay of long-acting thyroid stimulator (L.A.T.S.) ; the dose-response relationship. J. clin. Endocr. 1961, 21, 799.
9. McKenzie, J. M. Delayed thyroid response to serum from thyrotoxic patients. Endocrinology 1958, 62, 865.

10. McKenzie, J. M. Further evidence for a thyroid activator in hyperthyroidism. J. clin. Endocr. 1958, $20,380$.

11. McKenzie, J. M. Studies on the thyroid activator of hyperthyroidism. J. clin. Endocr. 1961, 21, 635.

12. McKenzie, J. M., and J. Fishman. Effects of antiserum in bioassay of thyrotropin and thyroid activator of hyperthyroidism. Proc. Soc. exp. Biol. (N. Y.) 1960, 105, 126.

13. Adams, D. D., H. D. Purves, and N. E. Sirett. The response of hypophysectomized mice to injections of human serum containing long-acting thyroid stimulator. Endocrinology 1961, 68, 154.

14. Berson, S. A., and R. S. Yalow. Quantitative aspects of iodine metabolism. The exchangeable organic iodine pool, and the rates of thyroidal secretion, peripheral degradation, and fecal excretion of endogenously synthesized organically bound iodine. J. clin. Invest. 1954, 33, 1533.

15. McKenzie, J. M. The bioassay of thyrotropin in serum. Endocrinology 1958, 63, 372.

16. Bakke, J., N. Lawrence, and S. Roy. Disappearance rate of exogenous thyroid-stimulating hormone (TSH) in man. J. clin. Endocr. 1962, 22, 352.

17. Randall, J. E. Elements of Biophysics, 2nd ed. Chicago, Year Book Medical Publishers, 1962, p. 111.

18. Utiger, R. D. The radioimmunoassay of plasma thyrotropin in hyperthyroidism and hypothyroidism (abstract). J. clin. Lab. Med. 1964, 64, 1013.

19. Björkman, S. E., T. Denneberg, and I. Hedenskog. Clinical evaluation of the thyroid stimulating hormone activity in exophthalmos. Acta endocr. (Kbh.) 1961, 38, 577.

20. Meek, J. C., A. E. Jones, U. J. Lewis, and W. P. VanderLaan. Characterization of the long-acting thyroid stimulator of Graves' disease. Proc. nat. Acad. Sci. (Wash.) 1964, 52, 342.

\section{SPECIAL NOTICE TO SUBSCRIBERS}

Post Offices will no longer forward the Journal when you move.

Please notify The Journal of Clinical Investigation, Business Office, 10 Stoughton Street, Boston, Mass. 02118, at once when you have a change of address, and do not omit the Zip Code number. 